\title{
Quaternionic Wigner-Ville distribution of analytical signal in hyperspectral imagery
}

\author{
Yang LIU \\ Shanghai Medical Instrumentation College \\ 101 Yingkou Road, Yangpu.District 200093 \\ Shanghai.China
}

\author{
Robert GOUTTE \\ University of Lyon,INSA, labo CREATIS \\ Umr Cnrs 5220,Inserm 1044 \\ Lyon,France
}

\begin{abstract}
The 2D Quaternionic Fourier Transform (QFT), applied to a real 2D image, produces an invertible quaternionic spectrum. If we conserve uniquely the first quadrant of this spectrum, it is possible, after inverse transformation, to obtain, not the original image, but a 2D quaternion image, which generalize in $2 \mathrm{D}$ the classical notion of $1 \mathrm{D}$ analytical image.
\end{abstract}

From this quaternion image, we compute the corresponding correlation product, then, by applying the direct QFT, we obtain the 4D Wigner-Ville distribution of this analytical signal. With reference to the shift variables $\chi_{1}, \chi_{2}$ used for the computation of the correlation product, we obtain a local quaternion WignerVille distribution spectrum.

Keywords-Analytical; signal; hyperspectral imagery; quaternionic distribution

\section{INTRODUCTION}

The most common method of analysis of the frequency content of an n-D real signal is the classical complex Fourier transform. The Fourier transforms have been widely used in signal and image processing. ever since the discovery of the Fast Fourier Transform in 1965 (Cooley-Tukey algorithm) which made the computation of Discrete Fourier Transform feasible using a computer.

Based on the concept of quaternion the quaternion Fourier transform (QFT) has been introduced by Ell (Ref 1) and implemented by Pei (Ref 2) with conventional 2D Fourier transform .

The analytic signal is a complex extension of a 1D signal that is based upon the Hilbert transform; it was introduced to signal theory by Gabor in 1946 (Ref 3).This representation gives access to the instantaneous amplitude and phase. Several attempts to generalize the analytic signal to two dimensions have been reported in the literature, based on the properties of Hilbert and Riesz transforms (Ref 4).

\section{CONCEPT OF QUATERNION'S NUMBERS}

The Quaternion, discovered by Hamilton (Ref 5) in 1843, also called hyper complex numbers, are the generalization of complex numbers. A complex number has two components: the real and the imaginary part.

The quaternion has four components:

$$
q=q_{r}+q_{i} \cdot i+q_{j} \cdot j+q_{k} \cdot k
$$

and $i, j, k$ obeys the rules as below:

$$
\begin{array}{cc}
i^{2}=j^{2}=k^{2}=-1 \\
i \cdot j=k & j \cdot i=-k \\
j \cdot k=i & k \cdot j=-i \\
k \cdot i=j & i \cdot k=-j
\end{array}
$$

We clearly observe that multiplication is associative and distributive, compared to addition, but not commutative.

When the real part is null, the corresponding number is called a pure quaternion. For a quaternion-valued function, we can define its magnitude as follow:

like its conjugate as:

$$
|q|=r=\sqrt{q_{r}^{2}+q_{i}^{2}+q_{j}^{2}+q_{k}^{2}}
$$

$$
\mathrm{q} *=\mathrm{q}_{\mathrm{r}}-\mathrm{q}_{\mathrm{i}} \mathrm{i}-\mathrm{q}_{\mathrm{j}} \mathrm{j}-\mathrm{q}_{\mathrm{k}} \mathrm{k}
$$

\section{2D QUATERNIONIC FOURIER TRANSFORM (QFT)}

The classic n-D complex Fourier transform is:

$$
F_{c}(\mathbf{u})=\int_{\mathfrak{R}^{n}} f(x) \prod_{i=1}^{i=n} \exp ^{-j 2 \pi u_{i} x_{i}} d x^{n}
$$

The QFT is based on the quaternion's concept, the quaternion Fourier Transform of a $2 \mathrm{D}$ real signal $f(x, y)$ is defined as:

$$
\mathrm{QFT}=F_{q}(u, v)=\int_{-\infty}^{+\infty} \int_{-\infty}^{+\infty} e^{-i 2 \pi u x} f(x, y) e^{-j 2 \pi v y} d x d y
$$

This QFT, of type 1 , is noted two-side. If the input $f(x, y)$ is a quaternion function and not only a real function, we can decompose $f(x, y)$ as:

$$
f(x, y)=f_{r}(x, y)+i . f_{i}(x, y)+f_{j}(x, y) j .+f_{k}(x, y) \mathrm{k} .
$$

where $f_{r}(x, y), f_{i}(x, y), f_{j}(x, y)$ and $f_{k}(x, y)$ are real functions. We obtain

$$
F_{q}(u, v)=F_{r q}(u, v)+i F_{i q}(u, v)+j F_{j q}(u, v)+k F_{k q}(u, v)
$$

The QFT is not identical to the 2D Clifford Fourier Transform (Ref 6,7), since the signal $f_{\mathrm{CF}}$ is sandwiched between the two exponential functions rather than standing on their left side. However the two transforms are identical for real 2D signals.

The QFT is invertible and its inverse is expressed as: 


$$
\begin{gathered}
F_{q}(u, v)=F_{r q}(u, v)+i F_{i q}(u, v)+j F_{j q}(u, v)+k F_{k q} \\
f(x, y)=\int_{-\infty}^{+\infty} \int_{-\infty}^{+\infty} e^{i 2 \pi u x} F_{q}(u, v) e^{j 2 \pi v y} d u d v
\end{gathered}
$$

The discrete Quaternion Fourier Transform (DQFT) was introduced by Sangwine and Ell in year 2000 (Ref 7)

This transform has many different expression types. In this paper, we only use the type 1 of DQFT, which has the following expression (direct formulation):

$$
F_{q}(u, v)=\sum_{M=0}^{M-1} \sum_{N=0}^{N-1} e^{-i 2 \pi\left(\frac{x u}{M}\right)} f(x, y) e^{-i 2 \pi\left(\frac{y v}{N}\right)}
$$

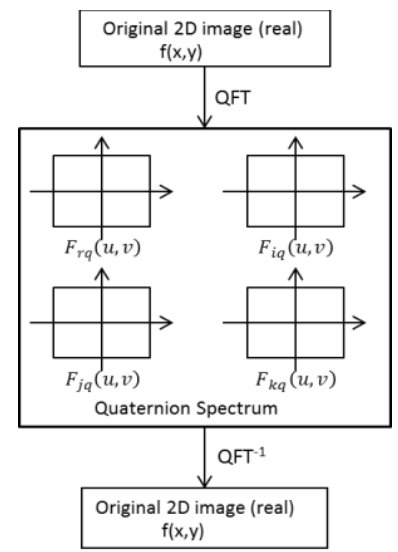

Fig. 1. Quaternion Fourier Transform Original image :
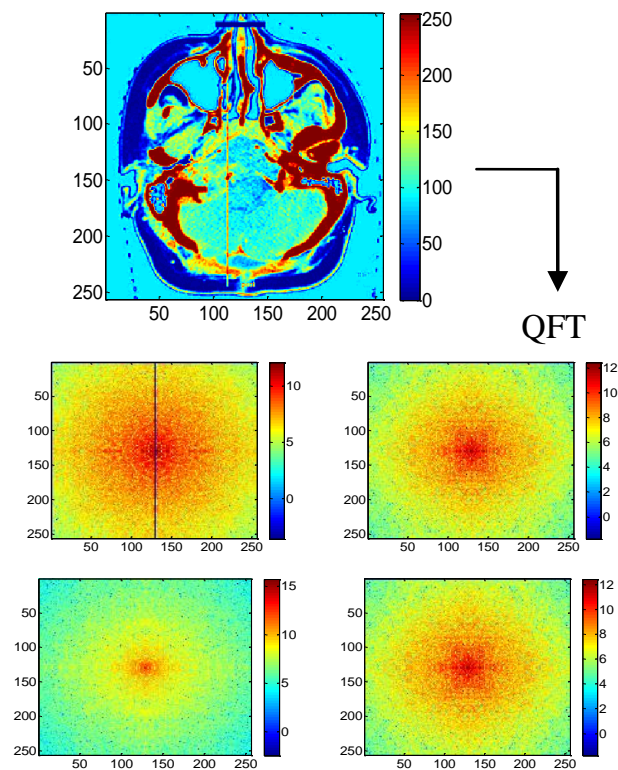

Fig. 2. Quaternionic spectrum

\section{PROPERTIES OF QUATERNIONIC SPECTRAL ANALYSIS: HERMITIAN SYMMETRY}

The quaternion spectrum QFT obeys the rules of the quaternion hermitian symmetry defined by the relations (Ref 8):

$$
\begin{gathered}
F_{q}(-u, v)=\propto_{j} \cdot F_{q}(u, v)=-j \cdot F_{q} \cdot j \\
F_{q}(-u,-v)=\propto_{k} \cdot F_{q}(u, v)=-k \cdot F_{q} \cdot k \\
F_{q}(u,-v)=\propto_{i} \cdot F_{q}(u, v)=-i \cdot F_{q} \cdot i
\end{gathered}
$$

where the functions $\propto_{i}, \propto_{j}$ and $\propto_{k}$ are called involutions of $F_{q}$

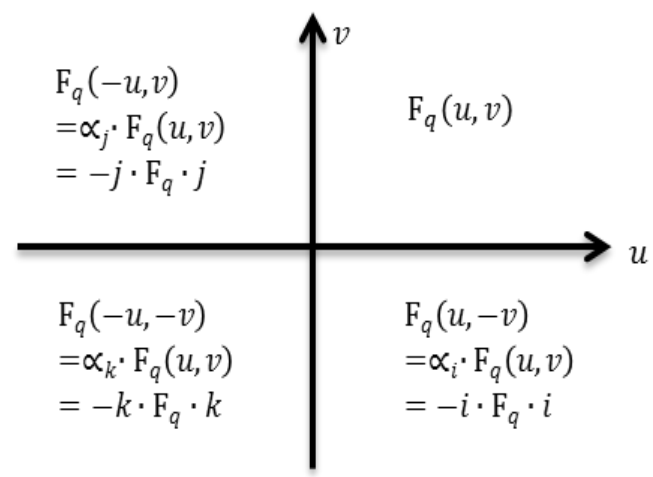

Fig. 3. hermitian properties of QFT

\section{DEFINITIONS AND ObTAINING Of ANALYTICAL SIGNAL}

Given a real $n-D$ signal $f(x)=f\left(x_{1}, x_{2, \ldots \ldots}, x_{n}\right)$

The n-D analytical signal with single orthant (the orthant is a half axis in 1-D, a quadrant in 2-D and an octant in3-D)

$$
\mathrm{f}_{1 \mathrm{q}}(\mathrm{x})=\mathrm{f}(\mathrm{x}) \otimes \otimes \otimes \ldots \psi^{\delta}(\mathrm{x})
$$

The symbol $\otimes \otimes \otimes \ldots$ denote the n-D convolution.

with: $\psi^{\delta}(\mathrm{x})=\prod_{i=1}^{i=n}\left[\delta \mathrm{x}_{1}+\frac{e_{i}}{\pi x_{i}}\right] \quad$ n-D hypercomplex delta distribution.

$$
\begin{aligned}
& \mathrm{f}_{1 \mathrm{q}}(\mathrm{x})=\mathrm{f}(\mathrm{x}) \otimes \otimes\left[\delta \mathrm{x}_{1}+\frac{e_{1}}{\pi x_{1}}\right]\left[\delta \mathrm{x}_{2}+\frac{e_{2}}{\pi x_{2}}\right] \\
& \mathrm{f}_{1 \mathrm{q}}(\mathrm{x})=\mathrm{f}(\mathrm{x}) \otimes \otimes\left[\delta \mathrm{x}_{1} \delta \mathrm{x}_{2}+\delta \mathrm{x}_{1} \frac{e_{2}}{\pi x_{2}}+\delta \mathrm{x}_{2} \frac{e_{1}}{\pi x_{1}}+\frac{e_{1}}{\pi x_{1}}\right. \\
& \left.\frac{e_{2}}{\pi x_{2}}\right]
\end{aligned}
$$

The analytical notion can be extended to taking the quaternions in Clifford algebra which contains the elements :

with: $\quad e_{1}=i \quad e_{2}=j \quad e_{1} e_{2}=k$ 


$$
\begin{gathered}
\mathrm{f}_{1 \mathrm{q}}(\mathrm{x})=\mathrm{f}\left(\mathrm{x}_{1}, \mathrm{x}_{2}\right)+\mathrm{ih}_{1}+\mathrm{jh}_{2}+\mathrm{kh} \\
\mathrm{h}=\mathrm{f}(\mathrm{x}) \otimes \otimes \frac{1}{\pi x_{1}} \frac{1}{\pi x_{2}} \text { total Hilbert transform } \\
\mathrm{h}_{1}=\mathrm{f}(\mathrm{x}) \otimes \otimes \delta \mathrm{x}_{2} \frac{1}{\pi x_{1}} \text { and } \\
\mathrm{h}_{2}=\mathrm{f}(\mathrm{x}) \otimes \otimes \delta \mathrm{x}_{1} \frac{1}{\pi x_{2}} \text { partial Hilbert transforms }
\end{gathered}
$$

In the spatial plane $f_{1 q}(x)$ is a quaternion which the four images are $f(x), h_{1}, h_{2}$ and $h$,represented in the fig 5 .

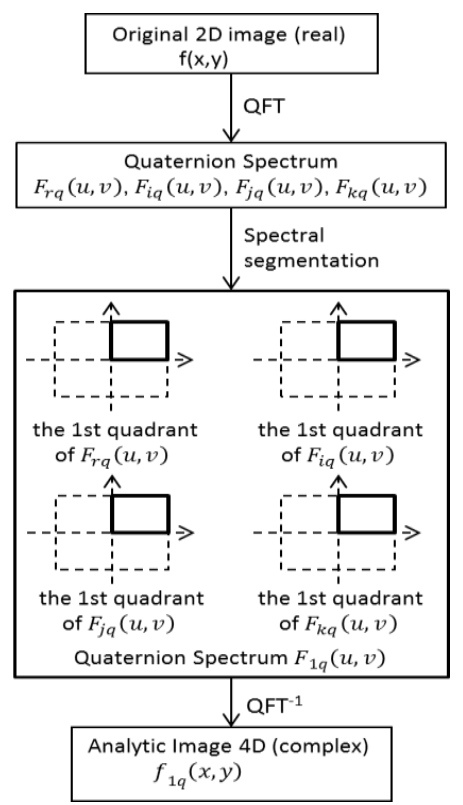

Fig. 4. Obtaining analytic image
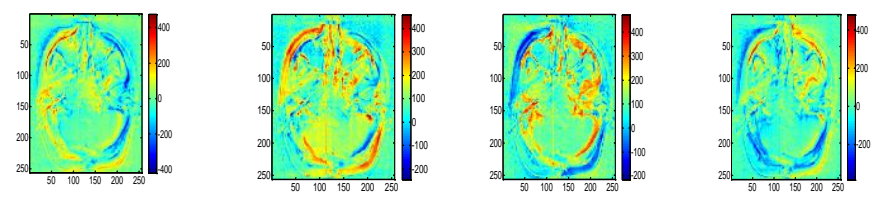

Real

i

$$
\begin{gathered}
W_{q}(x, y, u, v)=\iint e^{-i 2 \pi u \chi_{1}} \rho\left(x, y, \chi_{1}, \chi_{2}\right) e^{-j 2 \pi v \chi_{2}} d \chi_{1} d \chi_{2} \\
W_{q}(x, y, u, v)=W_{q r}(x, y, u, v)+\mathrm{i} \cdot \mathrm{W}_{\mathrm{qi}}(x, y, u, v) \\
+k \cdot W_{q k}(x, y, u, v)+j \cdot W_{q j}(x, y, u, v) \\
\rho(\mathrm{x}, \mathrm{y}, \mathrm{u}, \mathrm{v})=\mathrm{A}_{\mathrm{q}}+\mathrm{iB}_{\mathrm{q}}+\mathrm{j} \mathrm{C}_{\mathrm{q}}+\mathrm{kD}_{\mathrm{q}} \\
\mathrm{A}_{\mathrm{q}}=\mathrm{f}^{+} \mathrm{f}^{-}+\mathrm{h}_{1}^{+} \mathrm{h}_{1}^{-}+\mathrm{h}_{2}^{+} \mathrm{h}_{2}^{-}+\mathrm{h}^{+} \mathrm{h}^{-} \\
\mathrm{B}_{\mathrm{q}}=\mathrm{h}^{+} \mathrm{h}_{2}^{-}-\mathrm{f}^{+} \mathrm{h}_{1}^{-}+\mathrm{h}_{1}^{+} \mathrm{f}^{-}-\mathrm{h}_{2}^{+} \mathrm{h}^{-} \\
\quad \mathrm{C}_{\mathrm{q}}=-\mathrm{f}^{+} \mathrm{h}_{2}^{-}-\mathrm{h}^{+} \mathrm{h}_{1}^{-}+\mathrm{h}_{1}^{+} \mathrm{h}^{-}+\mathrm{h}_{2}^{+} \mathrm{f}^{-} \\
\mathrm{D}_{\mathrm{q}}=\mathrm{h}^{+} \mathrm{f}^{-}-\mathrm{f}^{+} \mathrm{h}^{-}+\mathrm{h}_{2}^{+} \mathrm{h}_{1}^{-}-\mathrm{h}_{1}^{+} \mathrm{h}_{2}^{-} \\
\mathrm{f}^{+}=\mathrm{f}\left(\mathrm{x}+0,5 \chi_{1}\right),\left(\mathrm{y}+0,5 \chi_{2}\right) \\
\mathrm{f}^{-}=\mathrm{f}\left(\mathrm{x}-0,5 \chi_{1}\right),\left(\mathrm{y}-0,5 \chi_{2}\right)
\end{gathered}
$$

with the same for the other functions $h_{1}, h_{2}$ and $h$.

$$
\begin{gathered}
W_{q}(x, y, u, v)=\iint_{+k D q) e^{-j 2 \pi v \chi_{2}} d \chi_{1} d \chi_{2}} e^{-i 2 \pi u \chi_{1}}(A q+i B q+j C q \\
W_{q}(x, y, u, v)=\iint_{+k D q) e^{-j \alpha 2} d \chi_{1} d \chi_{2}} e^{-i \alpha 1}(A q+i B q+j C q \\
+k \chi_{2}
\end{gathered}
$$

In the spectral space, the quaternionic Wigner distribution is a 4-D quaternion function :

$$
\begin{aligned}
& W_{\mathrm{qr}}(x, y, u, v)=\iint(A q \cos \alpha 1 \cos \alpha 2+B q \sin \alpha 1 \cos \alpha 2+C q \cos \alpha 1 \sin \alpha 2 \\
& +D q \sin \alpha 1 \sin \alpha 2) d \chi_{1} d \chi_{2} \\
& W_{\mathrm{qi}}(x, y, u, v)=\iint(B q \cos \alpha 1 \cos \alpha 2-A q \sin \alpha 1 \cos \alpha 2+D q \cos \alpha 1 \sin \alpha 2 \\
& -C q \sin \alpha 1 \sin \alpha 2) d \chi_{1} d \chi_{2} \\
& W_{\mathrm{qj}}(x, y, u, v)=\iint(C q \cos \alpha 1 \cos \alpha 2+D q \sin \alpha 1 \cos \alpha 2-A q \cos \alpha 1 \sin \alpha 2 \\
& -B q \sin \alpha 1 \sin \alpha 2) d \chi_{1} d \chi_{2} \\
& W_{\mathrm{qk}}(x, y, u, v)=\iint(D q \cos \alpha 1 \cos \alpha 2-C q \sin \alpha 1 \cos \alpha 2-B q \cos \alpha 1 \sin \alpha 2 \\
& +A q \sin \alpha 1 \sin \alpha 2) d \chi_{1} d \chi_{2}
\end{aligned}
$$

(this equation: $\quad \mathrm{W}_{\mathrm{q}}=\mathrm{W}_{\mathrm{qr}}+\mathrm{iW}_{\mathrm{qi}}+\mathrm{jW}_{\mathrm{qj}}+\mathrm{kW}_{\mathrm{qk}}$ holds for monogenic signals changing the subscripts $q$ to $\mathrm{M}$ )

In the particular case $\mathrm{u}=0$ and $\mathrm{v}=0$

$$
\mathrm{W}_{\mathrm{qr}}(\mathrm{x}, \mathrm{y}, 0,0)=\iint A q d \chi_{1} d \chi_{2}
$$

$$
\mathrm{W}_{\mathrm{qr}}(\mathrm{x}, \mathrm{y})=\iint\left[\mathrm{f}^{+} \mathrm{f}^{-}+\mathrm{h}_{1}^{+} \mathrm{h}_{1}^{-}+\mathrm{h}_{2}^{+} \mathrm{h}_{2}^{-}+\mathrm{h}^{+} \mathrm{h}^{-}\right] d \chi_{1} d \chi_{2}
$$

The local spectrum, centered on $\mathrm{u}=0$ and $\mathrm{v}=0$, is the sum of four spectra obtained from four correlation products, corresponding to the four components of the quaternionic analytic signal. 
VII. GENERALIZATION FOR 3-D REAL SIGNAL. For $\mathrm{n}=3$, the hypercomplex delta distribution $\psi^{\delta}(\mathrm{x})$ become:

$$
\psi^{\delta}\left(\mathrm{x}_{1}, \mathrm{x}_{2,} \mathrm{x}_{3}\right)=\delta \mathrm{x}_{1} \delta \mathrm{x}_{2} \delta \mathrm{x}_{3}+\left[\delta \mathrm{x}_{1}+\frac{e_{1}}{\pi x_{1}}\right]\left[\delta \mathrm{x}_{2}+\frac{e_{2}}{\pi x_{2}}\right]\left[\delta \mathrm{x}_{3}+\right.
$$

$\left.\frac{e_{3}}{\pi x_{3}}\right]$

and with the rules of Cayley-Dickson (Ref 9,11)

$$
\begin{aligned}
& \psi^{\delta}\left(\mathrm{x}_{1,} \mathrm{x}_{2}, \mathrm{x}_{3}\right)=\delta \mathrm{x}_{1} \delta \mathrm{x}_{2} \delta \mathrm{x}_{3}+\mathrm{e}_{1} \frac{\delta x_{2} \delta x_{3}}{\pi x_{1}}+\mathrm{e}_{2} \frac{\delta x_{1} \delta x_{3}}{\pi x_{2}}+ \\
& e_{3} \frac{\delta x_{3}}{\pi^{2} x_{1} x_{3}}+\mathrm{e}_{4} \frac{\delta x_{1} \delta x_{2}}{\pi x_{3}}+e_{5} \frac{\delta x_{2}}{\pi^{2} x_{1} x_{3}}+e_{6} \frac{\delta x_{1}}{\pi^{2} x_{1} x_{3}}+ \\
& e_{7} \frac{1}{\pi^{3} x_{1} x_{2} x_{3}}
\end{aligned}
$$

which is a octonion structure

$$
\begin{gathered}
\mathrm{f}_{\mathrm{CD}}\left(\mathrm{x}_{1}, \mathrm{x}_{2}, \mathrm{x}_{3}\right)=\mathrm{f}\left(\mathrm{x}_{1}, \mathrm{x}_{2}, \mathrm{x}_{3}\right) \otimes \otimes \otimes\left[\delta \mathrm{x}_{1} \delta \mathrm{x}_{2} \delta \mathrm{x}_{3}+\mathrm{e}_{1} \frac{\delta x_{2} \delta x_{3}}{\pi x_{1}}\right. \\
+\mathrm{e}_{2} \frac{\delta x_{1} \delta x_{3}}{\pi x_{2}}+e_{3} \frac{\delta x_{3}}{\pi^{2} x_{1} x_{3}}+\mathrm{e}_{4} \frac{\delta x_{1} \delta x_{2}}{\pi x_{3}}+e_{5} \frac{\delta x_{2}}{\pi^{2} x_{1} x_{3}}+ \\
\left.e_{6} \frac{\delta x_{1}}{\pi^{2} x_{1} x_{3}}+e_{7} \frac{1}{\pi^{3} x_{1} x_{2} x_{3}}\right]
\end{gathered}
$$

which corresponds to 8 images in the spectral plan: one real and seven imaginaries.

The process to obtain the Wigner-Ville distribution is the same as in the previous case $n=2$, but the computational complexity is increased by the passage, for the analytic signal, of an quaternionic structure to an octonion.

\section{CONCLUSION}

The use of the Fourier quaternionic transformation, associated at the convolution with the n-D delta distribution ,allows to obtain, from real image signal, a spatially hyper complex representation of the analytical signal (quaternion in 2D and octonion in 3D).From this approach, we can generalize the concept of the Wigner Ville distribution and obtain an analytical. tool with both a frequency selectivity and spatial localization.

Interesting applications can be envisaged in imagery, especially for the segmentation problems or in texture analysis (Ref 12).

\section{REFERENCES}

[1] T.A.ELL,Hypercomplex spectral transforms, $\mathrm{PhD}$ Thesis ,Université of Minnesota, 1992.

[2] S.C.Pei,J.J.Ding,J.H.Chang,Efficient Implementation of quaternion Fourier transform,IEEE transactions on signal processing,2001,vol49,no11,p.2783-2797.

[3] D.Gabor, Theorie of communication :Inst Elec.Eng.London,vol.3,pp:429-457,1946.

[4] M.Unser, Hilbert-Riesz wavelet Transform, Seventh Workshop on Information Optics, Fr, June 1-5, 2008

[5] W.R.Hamilton, On a new Species of imaginary quantities connected with a theory of Quaternion (1843),Meeting of the Royal Irish Academy.

[6] W.K.Clifford. Application of Grassmann's extensive algebra,Am.J.Math,1,350-1878.

[7] P.R.Girard, .Delachartre,P.Clarysse,R.Goutte,Mise en oeuvre du signal analytique dans les algèbres de Clifford, Colloque 23éme Gretsi,5-8 sept 2011,Bordeaux.

[8] S.I.Sangwine and T.A.Tell, The discrete Fourier transform of a colour image,Image Processing,Math Methods,Algorithms and Applications,pp:430-441,Chichester,2000.

[9] S.L.Hahn,Multidimensional Complex Signals with Single Orthant, Proceeding IEEE,vol80,pp1287-1300 August1992.

[10] S;L.Hahn,Wigner Quaternionic and Monogenic Signal ,IEEE Trans on Signal Processing,vol53, No 8, August 2005.

[11] K.M.Snopek,The study of properties of $\mathrm{nD}$ Analytic Signals,Radioengineering,vol 1,No1,April 2012

[12] Y.Zhu,R.Goutte,M.Amiel,On the use of a two-dimensional Wigner Ville distribution for texture segmentation.Signal Proc.30,205-220,1993. 